Original Research Article

\title{
Prescribing trends of anti glaucoma medication usage in treatment naive patients of primary open angle glaucoma in a tertiary care hospital in Eastern India
}

\author{
Aditi Maitra1, Shashwat Bhattacharyya $^{2 *}$, Shatavisa Mukherjee ${ }^{1}$, Nikhil Era ${ }^{3}$, \\ Sambuddha Ghosh ${ }^{2}$, Santanu Kumar Tripathi ${ }^{1}$
}

\begin{abstract}
${ }^{1}$ Dept of Clinical and
Experimental Pharmacology,

Calcutta School of Tropical

Medicine, Kolkata, West

Bengal, India

${ }^{2}$ Department of Ophthalmology, Calcutta National Medical

College, Kolkata, India

${ }^{3}$ Department of Pharmacology,

MGM Medical College,

Kishangunj, Bihar, India
\end{abstract}

Received: 13 March 2018

Accepted: 07 April 2018

*Correspondence to:

Dr. Shashwat Bhattacharyya,

Email: bhattacharyya.shashwat

@gmail.com

Copyright: () the author(s), publisher and licensee Medip Academy. This is an openaccess article distributed under the terms of the Creative Commons Attribution NonCommercial License, which permits unrestricted noncommercial use, distribution, and reproduction in any medium, provided the original work is properly cited.

\begin{abstract}
Background: Primary open angle glaucoma remains a high magnitude healthcare problem due to its prevalence and chronicity. The real world scenario of anti glaucoma medical therapy needs periodical auditing as far as drug prescriptions are concerned for formulation of treatment guidelines. Few studies from India have taken a longitudinal approach in this respect. This study was undertaken to identify such lacunae.

Methods: This was an open label, prospective, observational study. Each treatment naive patient was followed up for a period of 6 months, where their prescriptions were scanned for the type of the anti glaucoma medications, total number of medications, route of administration, their duration of use and their frequency of dosage and change in medications if any, in each visits.

Results: A gradual shift from monotherapy towards combination therapy was observed. Overall averages for prescriptions were topped by monotherapy at 44.25 followed by fixed dose combinations at 38.25 , and then combinationpolytherapy at 36 and the least number of prescriptions were for concurrent polytherapy at 17.5. Amongst all monotherapy agents, beta blockers were the most frequently prescribed drugs at baseline but their share of prescriptions was almost halved at the end of our study. The prostaglandin analogs on the other hand saw an upsurge in prescriptions from their baseline to the end of this study. Prostaglandin analogs and beta blockers as fixed dose combination was a popular prescription over the entire study duration.

Conclusions: Prescribing trends were remarkable for the shift from primary monotherapy towards fixed dose combinations as the study concluded. The decline in the number of beta blocker prescriptions and a rise in prostaglandin analog prescriptions is a testament to their better effectiveness and tolerability. The future of open angle glaucoma pharmacotherapy lies in prescribing more efficacious drugs either in monotherapy or in fixed combinations.
\end{abstract}

Keywords: Anti-glaucoma medications, Glaucoma management, Prescribing trends, Primary open angle glaucoma

\section{INTRODUCTION}

Glaucoma, a common and potentially blinding ocular disease having multifactorial etiology, is characterized by progressive acquired loss of retinal ganglion cells leading to optic nerve atrophy and visual field deficits. An estimated 60.5 million people suffer from open-angle and angle-closure glaucoma, which will be increasing to 79.6 million by $2020 .{ }^{1}$ In India, it is the leading cause of treatable non-reversible blindness. Primary open angle glaucoma (POAG) is estimated to affect about 6.48 million. ${ }^{2}$ India has a rapidly growing ageing population and this figure will increase to 16 million by 2020 . Glaucoma is a chronic disease for which lifelong control of intraocular pressure (IOP) is mandatory. The main modality of treatment is anti glaucoma medications. Medicines are thought to be safer; however, patients often need more than one medication to reach the "target" IOP, 
which may inadvertently increase the possibility of side effects, as well as long-term costs. In developed countries, the cost of glaucoma therapy is largely borne by government schemes or medical insurance companies while there is scarcity of studies on the cost of glaucoma therapy in developing countries. ${ }^{3}$ The number of available agents for the medical treatment of glaucoma has expanded greatly. Years ago, the choice was largely limited to miotics, epinephrine, and oral carbonic anhydrase inhibitors only.

Introduction of topical beta blockers in 1970s posed a milestone advancement. Gradually, topical carbonic anhydrase inhibitors, alpha-adrenergic agonists, and prostaglandin analogs came to limelight of treatment modalities which effectively lowered intraocular pressure (IOP) with milder side-effect profiles thus proving to be advantageous for the majority of patients.

With the introduction of innovative tools for early diagnosis and newer medications for treatment, diagnosis and management of glaucoma has become more complex. The newer glaucoma medications have advantages like superior efficacy, reduced dosing frequency, and better side effect profile. Owing to their superior efficacy and systemic safety, the prostaglandin analogues soon became the preferred choice for initial therapy in POAG patients.

The availability of combination therapy has also been an important upgrade in the fight against POAG. ${ }^{4}$ This study endeavoured to analyse the temporal trends in the prescription of medication for effective management of POAG during the study period.

\section{METHODS}

This was an open label, prospective, observational study. Enrolment of patients was done after obtaining approval from the institutional clinical research ethics committees and was carried out for a period of 6 months. Each patient was followed up for a period of 6 months.

\section{Inclusion criteria}

The study included all newly diagnosed patients of POAG (Age $\geq 40$ years) belonging to either genders receiving any type and any number of anti glaucoma medication. POAG was defined as mean IOP greater than $21 \mathrm{mmHg}$, with typical glaucomatous optic disc changes and demonstrable visual field defects with reliability indices within acceptable limits along with wide open angles on gonioscopy. Visual acuity $\geq 6 / 60$ (20/200) was considered included. Those able to give consent for participation and well comprehend the pre-tested questionnaires were considered for the study.

\section{Exclusion criteria}

The study excluded those having pre-existing hyperemia and dry eye disease, history of any ocular surgery or laser procedure, patients suffering from any other form of glaucoma, ocular inflammation or infection in the preceding 3 months, diabetes mellitus, hypertension, pregnant or lactating women, patients those are sensitive to preservatives, any other concomitant eye drops like lubricants or corticosteroids and one eyed patients. Concurrent use of other ophthalmic medications, contact lenses, conditions precluding Goldmann applanation tonometry and uncooperative patients were also excluded.

All consecutive treatment naïve patients newly diagnosed with POAG, meeting all inclusion criteria and willing to give informed consent were enrolled from the glaucoma clinic of the hospital. Prescriptions were scanned for patients receiving any of the anti glaucoma medications. Total number of medications, route of administration, their duration of use and their frequency of dosage and change in medications if any were noted. The type of therapy was also noted and grouped as follows

- Monotherapy: Therapy regimen involving a single drug for POAG,

- Combination therapy: Therapy regimen involving a single fixed dose combination (FDC) of two antiglaucoma drugs,

- Concurrent polytherapy: Therapy regimen involving 2 or more anti-glaucoma drugs prescribed separately,

- Combination polytherapy: Therapy regimen involving at least one FDC and one or more single drug along with.

\section{Statistical analysis}

Data collected was compiled and analyzed. For descriptive statistics, results were expressed in terms of means and percentages wherever applicable. All statistical calculations have been carried out using standard statistical software like Microsoft Excel and Statistical software for Social Sciences (SPSS) (Version 20).

\section{RESULTS}

A total of 68 patients diagnosed with primary open angle glaucoma and fulfilling the requisite inclusion criteria were included in the study after obtaining written informed consent. $60.29 \%(n=41)$ of the patients were male while rest were female. Mean age was found to be $58.16 \pm 9.35$ years, with the minimum age being 41 years and the maximum being 81years. Maximum number of the patients belonged to the age group of 61-70 years. Only 11 patients had familial history of glaucoma.

Analyzing the prescribing trends per visit (Table 1, Figure $1)$, the major observation showed gradual shift from monotherapy (46 to 38) towards combination therapy (34 to 44). Overall averages for prescriptions were topped by monotherapy at 44.25 followed by fixed dose combinations at 38.25, and then combination-polytherapy at 36 and the least number of prescriptions were for concurrent polytherapy at 17.5 . 
Table 1: Prescription types as per visit.

\begin{tabular}{|c|c|c|c|c|c|}
\hline & \multicolumn{4}{|c|}{ No. of Prescriptions n (\%) } & \multirow{2}{*}{ Mean No. of prescriptions } \\
\hline Type of Therapy & Visit 1 & Visit 2 & Visit 3 & Visit 4 & \\
\hline Monotherapy & $46(33.82)$ & $46(33.82)$ & $47(34.56)$ & $38(27.94)$ & 44.25 \\
\hline Combination Therapy & $34(25)$ & $38(27.94)$ & $37(27.21)$ & $44(32.35)$ & 38.25 \\
\hline Concurrent Polytherapy & $18(13.24)$ & $18(13.24)$ & $16(11.76)$ & $18(13.24)$ & 17.5 \\
\hline Combination Polytherapy & 38 (27.94) & $34(25)$ & $36(26.47)$ & $36(26.47)$ & 36 \\
\hline
\end{tabular}

Table 2: Overall distribution of drug groups at all visits.

\begin{tabular}{|c|c|c|c|c|c|c|c|c|c|c|c|c|c|c|}
\hline $\begin{array}{l}\text { Prescription } \\
\text { Type }\end{array}$ & Mon & herapy & $\begin{array}{l}\text { Com } \\
\text { ther }\end{array}$ & $\begin{array}{l}\text { ination } \\
\mathrm{y}\end{array}$ & & Con & Irren & polyt & rapy & & & $\begin{array}{l}\text { Comb } \\
\text { polytl }\end{array}$ & $\begin{array}{l}\text { natiol } \\
\text { rapy }\end{array}$ & \\
\hline VISIT NO & 前 & త્ઞ & 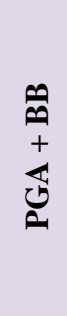 & 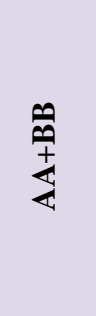 & $\begin{array}{l}\text { 苗 } \\
\text { 坖 }\end{array}$ & 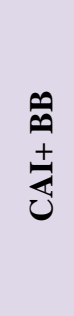 & 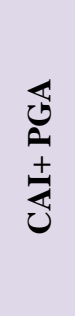 & 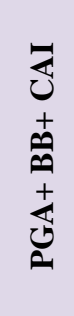 & 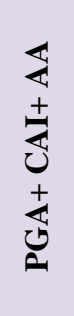 & 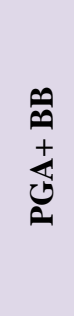 & 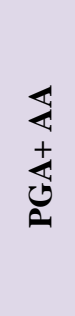 & 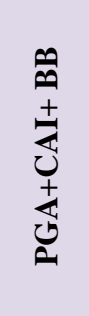 & 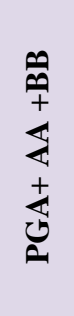 & 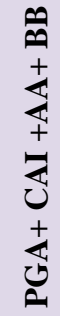 \\
\hline Visit 1 (Baseline) & 28 & 18 & 20 & 11 & 03 & 06 & 02 & 04 & 02 & 04 & 0 & 24 & 10 & 04 \\
\hline Visit $2\left(1^{\text {st }}\right.$ Follow up $)$ & 28 & 18 & 24 & 11 & 03 & 06 & 02 & 04 & 02 & 04 & 0 & 24 & 10 & 0 \\
\hline Visit 3 ( $2^{\text {nd }}$ Follow up) & 21 & 26 & 23 & 12 & 02 & 06 & 0 & 04 & 0 & 04 & 02 & 26 & 10 & 0 \\
\hline Visit 4 (Final Follow up & 14 & 24 & 30 & 12 & 02 & 06 & 04 & 04 & 0 & 0 & 04 & 26 & 10 & 0 \\
\hline Aggregate & 91 & 86 & 97 & 46 & 10 & 24 & 08 & 16 & 04 & 12 & 06 & 100 & 40 & 04 \\
\hline
\end{tabular}

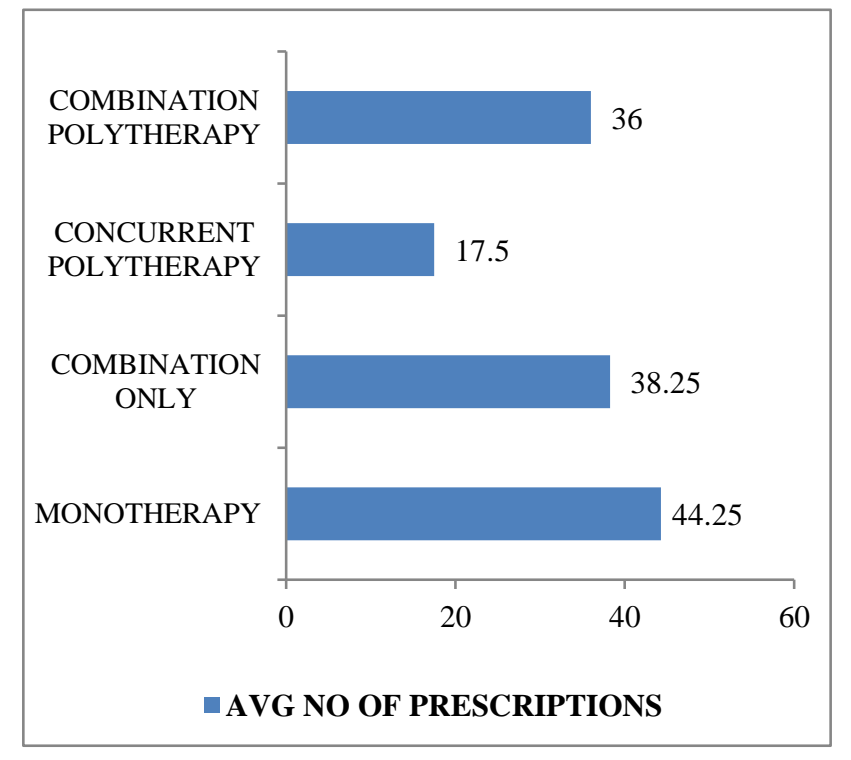

Figure 1: Average number of prescriptions as per therapy type.

Various anti-glaucoma drugs prescribed in this set-up included timolol and betaxolol as $\beta$ blockers, dorzolamide and acetazolamide as carbonic anhydrase inhibitors, bimatoprost, travoprost and latanoprost as prostaglandin analogues and brimonidine as alpha agonist respectively.
Overall distribution of all anti-glaucoma drugs in all visits were studied (Table 2). At baseline, the most frequently prescribed drugs for monotherapy in our study set-up were $\beta$ blockers $(n=28 ; 20.58 \%)$ followed by $P G$ analogues $(n=18 ; 13.23 \%)$. By the end of the study PGAs $(n=24$; $17.65 \%)$ had overtaken the BBs $(n=14 ; 10.29 \%)$, being the most prescribed monotherapy agent. Amongst FDCs, $\mathrm{PGA}+\mathrm{BB}$ were most frequently prescribed at baseline $(n=20 ; 14.71 \%)$ with figures even increasing at the final visit $(n=30,22.06 \%)$. Combination polytherapy regimen of PGA+BB+CAIs were the highest prescribed 3 drug combination both at baseline $(n=24 ; 17.64 \%)$ and at the final visit $(n=26 ; 19.12 \%)$. Amongst concurrent therapy, $\mathrm{CAI}+\mathrm{BB}$ was the highest prescribed regimen, being prescribed at a constant rate throughout the study $(n=6$; $4.41 \%)$.

\section{DISCUSSION}

Glaucoma describes a progressive optic neuropathy that is recognized by the appearance of characteristic cupping of the optic disc associated with corresponding visual deficit. The condition has as its basis gradual loss of retinal ganglion cells and their axons, and as a major risk factor, intraocular pressure (IOP). Glaucoma is progressive, and, if left unchecked, may cause blindness. Glaucoma is classified as primary if the cause of elevated IOP is unknown and secondary where a cause is known. They 
may again be congenital or in born and acquired or adult onset. Conventionally the term primary open-angle glaucoma (POAG) is applied to eyes with primary chronic glaucoma with open anterior chamber drainage angles and elevated IOP. Glaucoma is the second leading cause of blindness worldwide after cataract. In developed nations, $50 \%$ of those with glaucoma do not know they have it, a figure that rises to over $90 \%$ in developing nations with poor access to health-care. Global prevalence rates for POAG range from $1.4 \%$ to $4.5 \%$. India accounts for $12.9 \%$ of all POAG cases in the world. In a recently published study by Paul et al, which was the largest population-based glaucoma study in India to date, POAG was the most common form of glaucoma in the study population $(1.45 \%$ to $2.10 \%)^{5}$

The various pharmacological options available in the management of POAG include miotic medications, alpha adrenergic agonists, beta blockers, carbonic anhydrase inhibitors and prostaglandin analogs (PGAs). Amongst the anti- glaucoma medications, beta-blockers (timolol) and prostaglandins (latanoprost, bimatoprost and travoprost) have been found to be most effective in lowering the intraocular pressure (IOP). The PGA however have been demonstrated to have better efficacy than beta blockers. The effectiveness of these groups of drugs needs to be widely and repeatedly studied in order to provide inputs for future trends in the management of POAG.

The choices of drugs to be prescribed were governed by the target IOP, availability of drugs in the pharmacy, the side effect/contraindications of these drugs and the profile of the patients with respect to their ability to follow the prescribed regimen. The first line drugs like BBs and PGAs were supplemented with $2^{\text {nd }}$ line drugs like AAs and CAIs wherever the situation so demanded. None of the second line drugs were prescribed for monotherapy.

In the present study amongst all monotherapy agents, BB were the most frequently prescribed drugs at baseline $(n=28,20.58 \%)$ but their share of prescriptions was down to half of that number at the end of our study $(n=14$, $10.29 \%$ ). The PG analogs on the other hand saw an upsurge in prescriptions from their baseline figures $(n=18$, $13.23 \%)$ to the end of this study $(n=24,17.65 \%)$. PGA+BB combination was a popular prescription over the duration of the study, ranging from $20(14.71 \%)$ at baseline to 30 $(22.06 \%)$ at visit 4 . The highest prescribed three drug regimen was $\mathrm{PGA}+\mathrm{BB}+\mathrm{CAI}$ and their prescriptions remained steady till the end $(n=26,19.11 \%)$.

In a recent Taiwanese study by Lin et al, in 2015, it was observed that the number of ocular hypotensive drug (OHD) prescriptions has tripled during the preceding 11 years. ${ }^{6}$ Although the prescribing trend has moved away from monotherapy with $\beta$-blockers, this class remained the most commonly used OHD up to 2007. Prostaglandin analogs were the second most frequently prescribed OHDs since 2000. Changing prescribing patterns and increased patients were the main contributors to the rise in $\mathrm{OHD}$ expenditures. Part of increased expenditure was likely offset by reduced needs for laser and surgical interventions. An English study by Connor et al, studying the prescription trends from 2000 to 2012 found out that the prescriptions for prostaglandin analogues increased fourfold, while there was a threefold decrease in the use of beta blockers. ${ }^{7}$ The most commonly prescribed glaucoma medication was latanoprost. The introduction of generic latanoprost in 2012 more than halved the cost associated with this medication. In an earlier study by De Natale et al, it was seen that from 1997 to 2002 there was a sharp drop in the prescribing of beta-blockers (79\% in 1997 and 55\% in 2002). ${ }^{8}$ A marked increase in the use of prostaglandin derivatives (0\% in 1997 and $18 \%$ in 2002) was registered and a marked increase in the prescribing of carbonic anhydrase inhibitors (5\% in 1997 and 14\% in 2002) was also noted. From 1997 to 2002 there was a trend towards drug addition rather than substitution, so that the number of drugs used per patient increased.

A notable study from Indian scenario by Yadav et al, reported that $\beta$ blockers (93.88\%) were found to be the most frequently prescribed for POAG. ${ }^{9}$ Other groups included oral carbonic anhydrase inhibitors (26.66\%), topical carbonic anhydrase inhibitors (15\%), $\alpha-2$ adrenoceptor agonists (17.22\%), prostaglandin analogs (PGA; 8.88\%) and cholinergics (3.33\%). Timolol $(82.22 \%)$ was the most frequently prescribed drug and timolol with acetazolamide $(17.22 \%)$ was the most commonly prescribed drug combination. ${ }^{9}$ Fixed dose combinations constituted $26.66 \%$ of prescriptions. Another study by Rai et al, reported in their analysis that $64.86 \%$ patients used topical monotherapy, with a $\beta$ blocker $(41.66 \%)$, timolol $0.5 \%$ being the most common drug in the same group $(29.16 \%){ }^{10}$

The present study observed preponderance of topical medications as opposed to systemic drugs. Only 5 (7.35\%) prescriptions of oral acetazolamide in the first visit were noted. The rest $63(92.65 \%)$ prescriptions were of topical medications. Visits 2, 3 and 4 were exclusive for topical only drug prescriptions. The monotherapy prescriptions accounted for approximately $33 \%$ of the patients in the $1^{\text {st }}$, $2^{\text {nd }}$ and $3^{\text {rd }}$ visits while fixed dose combinations were 25 $27.94 \%$ for visits 1,2 and 3 . Thereafter monotherapy prescriptions fell to $27.94 \%$ while fixed dose combinations rose to $32.35 \%$ at visit 4 , clearly demonstrating a shift in the trend of prescriptions.

This is in contrast to Yadav et al, who recorded a higher percentage of monotherapy $(46.67 \%)$ and Rai et al, who reported $64.86 \%$ monotherapy prescriptions. FDC containing polytherapy regimens remained steady in terms of percentage throughout the study, bearing testimony to the fact that effectiveness, adherence and ocular surface disease are well managed by their use. Here the use of FDC was $32.35 \%$ by the end of the study which in contrast to Yadav et al, report of $26.66 \%$ is much higher. Concurrent polytherapy with separate drugs was the least popular regimen, consistently figuring at $11.76 \%$ to $13.24 \%$. 
Our study had certain limitations too. The limited study duration and small sample size may not have predicted any obvious shift in prescribing trends, though notable observations were analyzed. Moreover, being a government set-up, choice of prescribing may have been influenced by availability in hospital inventory. The study employed stringent exclusion criteria to increase the internal validity, which may have affected the sample size. The study being a single centre experience may not be a representative of national data.

The strength of the work lies in considering treatment naive, newly diagnosed cases that were not prone to any previous influences with respect to drug or surgical therapy, unlike majority of studies. This study remains the first of its kind from this part of the country, sparking a need of observing the prescribing trend in larger set-ups in other parts of the nation.

\section{CONCLUSION}

Prescribing trends were remarkable for the shift from primary monotherapy towards fixed dose combinations as the study concluded. Within monotherapy prescriptions themselves there was a decline in the number of beta blocker prescriptions and a rise in prostaglandin analog prescriptions. Concurrent polytherapy was the least prescribed regimen. The combination of beta blockers with prostaglandin analogs was the most prescribed regimen.

Funding: No funding sources

Conflict of interest: None declared

Ethical approval: The study was approved by the Institutional Ethics Committee

\section{REFERENCES}

1. Quigley HA, Broman AT. The number of people with glaucoma worldwide in 2010 and 2020. The British J Ophthalmol. 2006;90(3):262-7.

2. George R, Ve RS, Vijaya L. Glaucoma in India: estimated burden of disease. $\mathrm{J}$ Glaucoma. 2010;19:391-7.
3. Nayak B, Gupta S, Kumar G, Dada T, Gupta V, Sihota R. Socioeconomics of long-term glaucoma therapy in India. Ind J Ophthalmol. 2015;63(1):20-4.

4. Sambhara D, Aref AA. Glaucoma management: relative value and place in therapy of available drug treatments. Therapeutic Advances in Chronic Disease. 2014;5(1):30-43.

5. Paul C, Sengupta S, Choudhury S, Banerjee S, Sleath BL. Prevalence of glaucoma in Eastern India: The Hooghly River Glaucoma Study. Ind J Ophthalmol. 2016;64(8):578-83.

6. Lin JC. The use of ocular hypotensive drugs for glaucoma treatment: changing trend in Taiwan from 1997 to 2007. J Glaucoma. 2015;24(5):364-71.

7. Connor, A J, and S G Fraser. "Glaucoma Prescribing Trends in England 2000 to 2012." Eye 28.7. 2014:8639.

8. De Natale R, Draghi E, Dorigo MT. How prostaglandins have changed the medical approach to glaucoma and its costs: an observational study of 2228 patients treated with glaucoma medications. Acta Ophthalmol Scand. 2004;82(4):393-6.

9. Yadav AK, Patel V. Drug use in primary open angle glaucoma: A prospective study at a tertiary care teaching hospital. Ind J Pharmacol. 2013;45(2):117.

10. Rai S, Khilji S, Rao LG, Hegde P, Gonsalves SR, Shanbhag TV. Prescribing pattern and adverse events of drugs used in patients with primary open-angle glaucoma (POAG) attending a tertiary care hospital: Retrospective study. National J Physiol Pharmacy and Pharmacol. 2017;7(2):189-93.

Cite this article as: Maitra A, Bhattacharyya $S$, Mukherjee S, Era N, Ghosh S, Tripathi SK.

Prescribing trends of anti glaucoma medication usage in treatment naive patients of primary open angle glaucoma in a tertiary care hospital in Eastern India. Int J Basic Clin Pharmacol 2018;7:971-5. 